\title{
Investigation of the Flat-Beam Model of the Beam-Beam Interaction
}

\author{
Bjoern S. Schmekel, Georg Hoffstaetter and Joseph T. Rogers \\ Cornell University, Ithaca, New York 14853, USA
}

Report: Cornell CBN-03-12

PAC'03, Portland/OR

\begin{abstract}
At the interaction point of a storage ring collider each beam is subject to perturbations due to the electromagnetic field of the counter-rotating beam. For flat beams, a well known approximation models the beam by a current sheet which is uniform in the horizontal plane, restricting the particle motion to the vertical direction. In this classical model a water-bag beam distribution is used to find working points and beam-beam tune shift parameters which lead to a stable beam distribution. We try to find stability criteria for a more realistic Gaussian equilibrium distribution. In order to analyze the instabilities, a linearized Vlasov equation is solved computing radial and angular modes to first order in the displacement from the design trajectory.
\end{abstract}

\section{BEAM EVOLUTION}

We model the flat beam as a current sheet which is uniform in the horizontal direction, $x$, and consider only motion in the vertical direction, $y$. Consider one-dimensional phase space distributions $\psi_{1}$ and $\psi_{2}$ of the two beams which are normalized to unity. Then the impulse from the second (first) on the first (second) beam is

$$
\Delta y_{1,2}^{\prime}=-I_{\psi_{2,1}}(y, s)
$$

where we define

$I_{\psi}(y, s) \equiv \frac{4 \pi N r_{e}}{\gamma} \int_{-\infty}^{\infty} d \bar{y} \operatorname{sgn}(y-\bar{y}) \int_{-\infty}^{\infty} d \bar{y}^{\prime} \psi\left(\bar{y}, \bar{y}^{\prime}, s\right)$

and $N$ is the number of particles per unit width in $x$ and $r_{e}$ the classical radius of the electron. The equations describing the motion of $\psi_{1,2}$ are given by the two Vlasov equations

$$
\begin{array}{r}
\frac{\partial \psi_{1,2}}{\partial s}+y^{\prime} \frac{\partial \psi_{1,2}}{\partial y}-K(s) y \frac{\partial \psi_{1,2}}{\partial y^{\prime}}- \\
\frac{\partial \psi_{1,2}}{\partial y^{\prime}} \delta_{p}(s) I_{\psi_{2,1}}(y, s)=0
\end{array}
$$

We want to determine whether the beam is stable. That is, we want to know if small perturbations of the phase space density grow. Thus, we choose a perturbative ansatz

$$
\psi_{1,2}=\psi_{0} \pm \Delta \psi_{1,2}
$$

where $\psi_{0}$ is the equilibrium distribution, i.e. a solution of eqn. 3 with $\psi_{1}\left(y, y^{\prime}, s\right)=\psi_{2}\left(y, y^{\prime}, s\right)=\psi_{0}\left(y, y^{\prime}\right)$. Substituting eqn. 4 into eqn. 3 , subtracting eqn. 3 written for the equilibrium distribution and neglecting the term which is a product of two perturbations we find

$$
\begin{array}{r}
\frac{\partial \Delta \psi_{1,2}}{\partial s}+y^{\prime} \frac{\partial \Delta \psi_{1,2}}{\partial y}-\frac{\partial \Delta \psi_{1,2}}{\partial y^{\prime}} F(y, s)- \\
\delta_{p}(s) \frac{\partial \psi_{0}}{\partial y^{\prime}} I_{\Delta \psi_{2,1}}=0
\end{array}
$$

where

$$
F(y, s)=K(s) y+\delta_{p}(s) I_{\psi_{0}}(y)
$$

If we approximate the beam-beam force as linear in $y$

$$
F(y, s) \approx F(s) y
$$

we can treat the perturbation as a part of the perturbed focusing function $F(s)$. In the next step we transform eqn. 5 to action-angle coordinates

$$
y=\sqrt{2 \beta J} \cos \phi \quad y^{\prime}=-\sqrt{2 \beta J} \frac{\sin \phi+\alpha \cos \phi}{\beta}
$$

where $\beta$ denotes the beta function perturbed by the beta beat due to the linearized beam-beam kick from $\psi_{0}$. We choose a smooth approximation in which $\alpha=0$. Forming the linear combinations for the $\sigma$ - and the $\pi$-mode $f_{ \pm}=$ $\Delta \psi_{1} \pm \Delta \psi_{2}$, eqn. 5 can be decoupled and rewritten in action-angle coordinates as

$$
\frac{\partial f_{ \pm}}{\partial s}+\frac{1}{\beta} \frac{\partial f_{ \pm}}{\partial \phi} \pm \sqrt{2 \beta J} \sin \phi \delta_{p}(s) \frac{\partial \psi_{0}}{\partial J} I_{f_{ \pm}}=0
$$

assuming that $\psi_{0}=\psi_{0}(J)$. In order to stay consistent with our approximations we still have to linearize the remaining integral. In the following discussion we omit the label \pm .

\section{EQUILIBRIUM DISTRIBUTION}

When the interaction term in eqn. 3 is not considered any differentiable distribution which depends solely on $J$ is an equilibrium distribution. In general $\psi_{0}$ will be a function of both $J$ and $\phi$, though. Fortunately, an arbitrary differentiable function of $J$ is an equilibrium distribution at least to linear order in $y$ after introducing the perturbed betatron function. We choose a Gaussian equilibrium distribution

$$
\psi_{0}(J)=\frac{1}{2 \pi \epsilon} e^{-\frac{J}{\epsilon}}
$$

since in the presence of damping and quantum excitation the beam distribution naturally tends to a Gaussian distribution. 


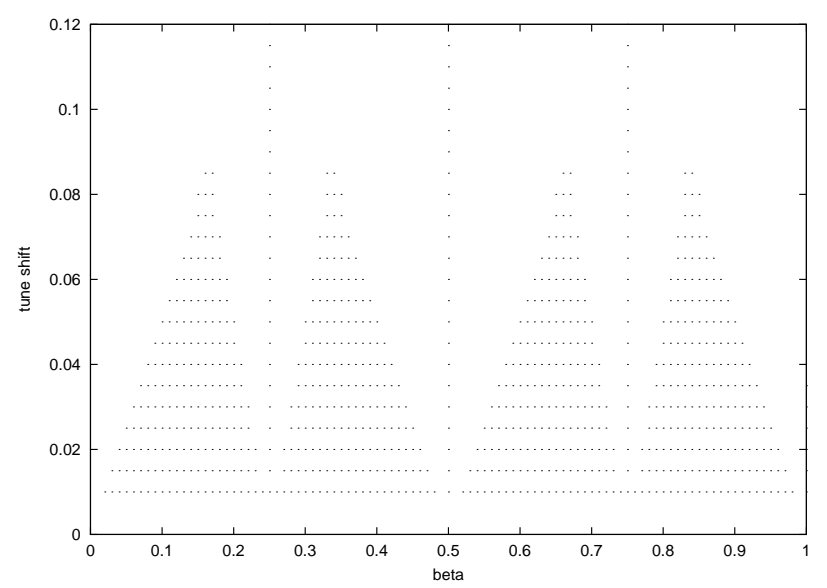

Figure 1: Stability diagram for $n=0, l=-2 \ldots 2$

\section{SOLVING THE EQUATIONS OF MOTION}

We expand the linearized version of eqn. 9 using the ansatz

$$
f(J, \phi, s)=\sum_{n^{\prime} l^{\prime}} g_{n^{\prime} l^{\prime}}(s) e^{-\frac{J}{\epsilon}} L_{n^{\prime}}\left(\frac{J}{\epsilon}\right) e^{i l^{\prime} \phi}
$$

where the n'-th Laguerre polynomial is denoted by $L_{n^{\prime}}$ and the summation runs from 0 to $\infty$ for $n^{\prime}$ and from $-\infty$ to $\infty$ for $l^{\prime}$. Substituting eqn. 10 and eqn. 11 into eqn. 9 we obtain eqn. 12

\section{COHERENT BEAM-BEAM INSTABILITY}

We solve the ODE 12 and rewrite the solution in matrix form such that the beam transport after one turn is described by a matrix $T$ which acts on a column vector $G$ that contains all $g_{n l}$, i.e. $G(C)=T G(0)$ and parametrize the beam-current by introducing the tune shift parameter

$$
\Delta \nu \equiv \frac{N r_{e}}{\gamma} \sqrt{\frac{2 \beta^{*}}{\pi \epsilon}}
$$

where $\beta^{*}$ denotes the beta function at the interaction point. In order to decide whether the system is stable or not we have to find out what happens to an arbitrary initial perturbation after a large number of turns, i.e. one needs to consider the limit $T^{N}$ where $N \longrightarrow \infty$. Every matrix norm of the latter quantity tends to infinity if the absolute value of all eigenvalues of $T$ are bigger than 1 .

\section{RESULTS AND DISCUSSION}

In Fig. 1 and 2 we have drawn a point if the absolute value of all eigenvalues of $T$ is smaller or equal 1 for both the $\sigma$ - and the $\pi$-mode. The first and second order resonances can be recognized clearly. Resonances of orders higher than 2 cannot be expected in our linearized model. From the diagrams we conclude that the inclusion of radial modes tends to stabilize the beam. In Fig. 3 and 4 we determine which mode becomes unstable by selecting

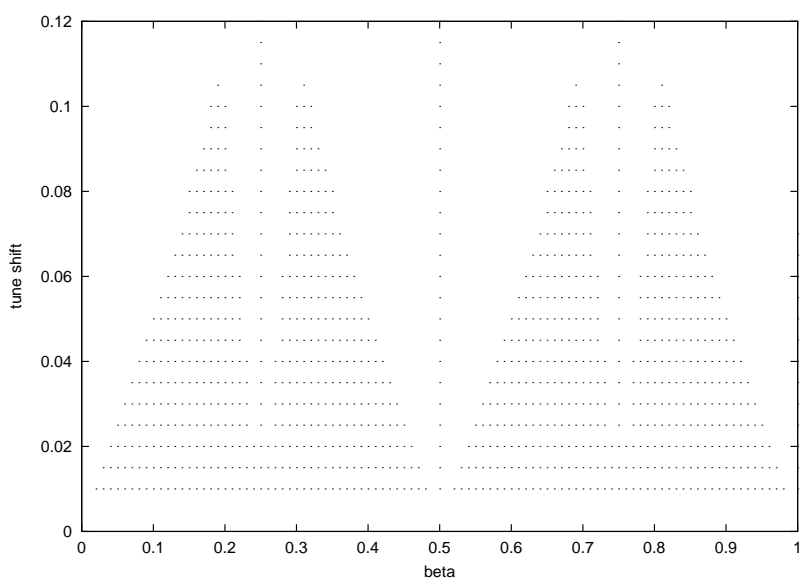

Figure 2: Stability diagram for $n=0 \ldots 2, l=-2 \ldots 2$

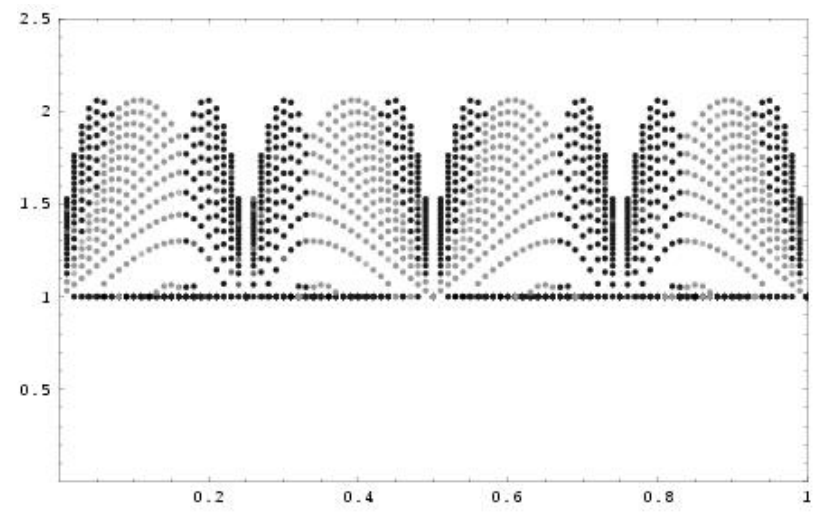

Figure 3: Absolute value of the largest eigenvalue vs. perturbed tune. Light grey points indicate unstable $l= \pm 1$ modes, medium grey and dark grey points indicate unstable $l= \pm 2$ modes. The following modes were included: $n=0, l=-2 \ldots 2$

the biggest component of the eigenvector which is associated with the largest eigenvalue. The plot shows that in the absence of dynamics in the radial direction $l= \pm 1$ and $l= \pm 2$ modes become unstable in the vicinity of $\nu=0.5$, but in Fig. 4 only $l= \pm 1$ modes are excited around $\nu=0.5$. Furthermore, the unstable $l= \pm 2$ modes which accumulate in the vicinity of $\nu=0.25$ and $\nu=0.75$ are attenuated. Therefore, the radial motion leads to a damping of the $l= \pm 2$ modes. In Fig. 5 we computed the phase of the largest eigenvalue of $l= \pm 2$ instabilities ( $\sigma$ mode only) versus the perturbed tune for various $\Delta \nu$. The slope of the two lower lines is 2 which indicates that the collective oscillation frequency of the quadrupole mode is twice the single particle oscillation frequency. In Fig. 6 the spread is significantly lower. In an unstable region the imaginary part of the highest eigenvalue vanishes which causes those characteristic plateaus. 


$$
\begin{gathered}
\frac{\partial g_{n l}}{\partial s}+\frac{i l}{\beta} g_{n l} \pm \frac{(2 n) ! \sqrt{\pi}}{2(2 n-1)\left(2^{n} n !\right)^{2}} \frac{4 \pi N r_{e}}{\gamma \epsilon^{2}} \frac{\sqrt{2 \beta \epsilon}}{2 \pi} \frac{1}{2 i}\left(\delta_{l, 1}-\delta_{l,-1}\right) \delta_{p}(s)\left[-4 \epsilon \sum_{l^{\prime}} g_{0,2 l^{\prime}+1} \frac{(-1)^{l^{\prime}}}{2 l^{\prime}+1}\right] \\
\pm \frac{2 \beta(-1)}{2 \pi} \frac{1}{4 i} \frac{4 \pi N r_{e}}{\gamma \epsilon}\left(\delta_{n, 0}-\delta_{n, 1}\right)\left(\delta_{l, 2}-\delta_{l,-2}\right) \delta_{p}(s)\left[2 \sqrt{\frac{2 \epsilon}{\beta}} \sum_{n^{\prime} l^{\prime}} g_{n^{\prime}, 2 l^{\prime}}(-1)^{l^{\prime}} \frac{\left(2 n^{\prime}\right) ! \sqrt{\pi}}{\left(2^{n^{\prime}} n^{\prime} !\right)^{2}}\right]=0
\end{gathered}
$$

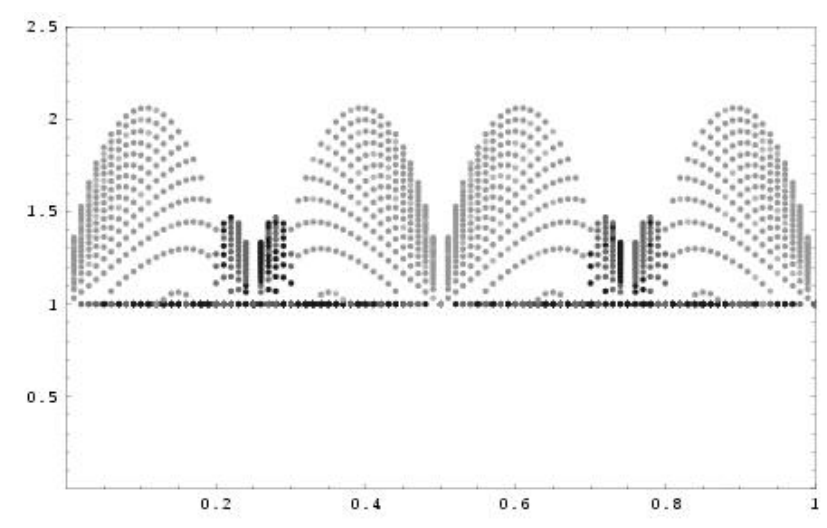

Figure 4: Same as Fig. 3, but for $n=0 \ldots 1, l=-2 \ldots 2$

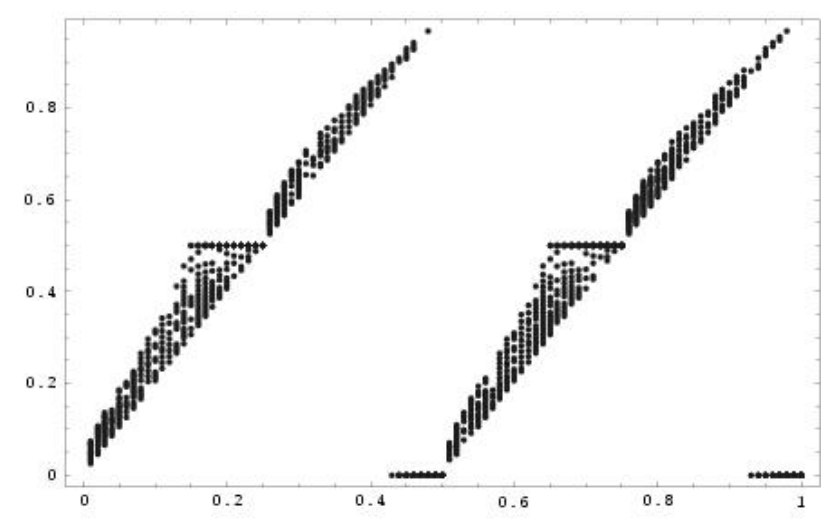

Figure 5: Phase vs. perturbed tune for $n=0, l= \pm 2$ modes ( $\sigma$-mode only).

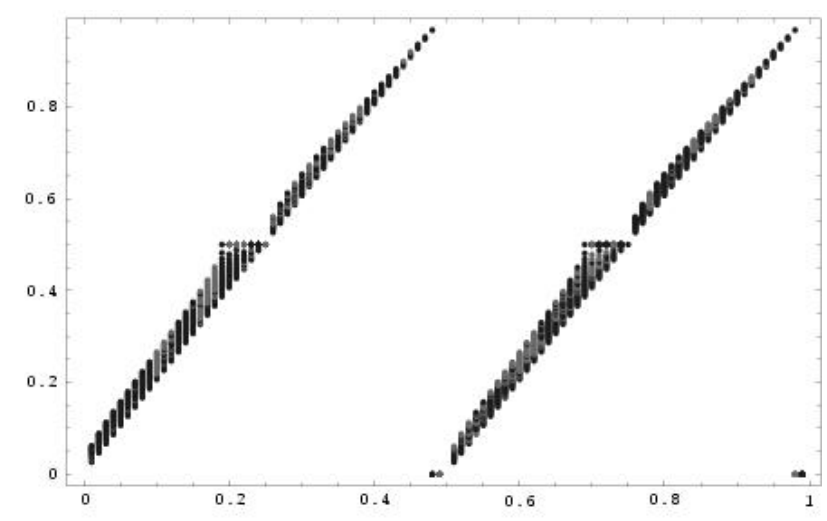

Figure 6: Phase vs. perturbed tune for $n=0 \ldots 1, l= \pm 2$ modes.

\section{POSSIBLE EXTENSIONS}

We have extended our model to account for damping by synchrotron radiation. In order to obtain the equilibrium distribution 10 quantum excitation must be included as well. This turns eqn. 3 into a Fokker-Planck equation. In our preliminary computations we found that the graphs we presented above remain unchanged for realistic values of the damping and excitation coefficients. To simplify the Fokker-Planck equation we averaged over the phases in the damping and excitation terms but not in the beam-beam interaction term. This can be justified since the betatron phases in the terms for damping and quantum excitation change during one turn while the phase in the interaction term changes only once per turn.

Higher order resonances can be studied by not linearizing the integral in eqn. 9 and assuming that $f_{ \pm}$contains only one radial mode. However, this procedure is complicated by the fact that eqn. 10 is not an equilibrium anymore. Ignoring these problems one can obtain plots similar to Fig. 1 with higher order resonances. For accelerators with different tunes for the rotating and counter-rotating beam a bigger transfer matrix which describes the evolution of the $g_{n l}$ for both beams can be derived easily.

\section{ACKNOWLEDGMENTS}

We would like to express our gratitude to Alex Chao from SLAC for his helpful criticism. This work was supported by the National Science Foundation.

\section{REFERENCES}

[1] A. W. Chao, R. D. Ruth, "Coherent Beam-Beam Instability in Colliding-Beam Storage Rings", Particle Accelerators, 1985, Vol. 16, pp. 201-216, Gordon and Breach 